\title{
Patient Perceptions of Robotic Surgery for Benign Gynecologic Disease
}

\author{
Vargas $\mathrm{MV}^{* 1}$, Cohen $\mathrm{SL}^{2}$, Jackson $\mathrm{TR}^{3}$, Hill-Lydecker $\mathrm{CI}^{2}$, Einarsson $\mathrm{JI}^{2}$ and Wang $\mathrm{KC}^{4}$ \\ ${ }^{1}$ Department of Obstetrics and Gynecology, Division of Gynecology, George Washington University Medical Center, \\ Washington DC, United States \\ ${ }^{2}$ Department of Obstetrics and Gynecology, Division of Minimally Invasive Gynecologic Surgery, Brigham and \\ Women's Hospital, Boston, MA, United States \\ ${ }^{3}$ Baylor Medical Center at Garland, Garland, TX, United States \\ ${ }^{4}$ Department of Obstetrics and Gynecology, The Minimally Invasive Pelvic Surgery Center, The Johns Hopkins Hos- \\ pital, Baltimore, MD, United States
}

*Corresponding author: Vargas MV, MD, Department of Obstetrics and Gynecology, Division of Gynecology, George Washington University Medical Center, 2150 Pennsylvania Ave, NW Suite 6a-408, Washington, DC 20037, United States, Tel: (240)432-8428, E-mail: vickyvar55@gmail.com

Citation: Vargas MV, Cohen SL, Jackson TR, Hill-Lydecker CI, Einarsson JI, et al. (2016) Patient Perceptions of Robotic Surgery for Benign Gynecologic Disease. J Gynecol Res 2(1): 101. doi: 10.15744/2454-3284.2.101

Received Date: Febuary 16, 2016 Accepted Date: October 26, 2016 Published Date: October 27, 2016

\begin{abstract}
Studies comparing robotic-assisted laparoscopic surgery (RALS) and conventional laparoscopic surgery (CLS) suggest that the two approaches have similar outcomes. However, RALS leads to higher cost and longer operative time. We sought to investigate patient perceptions of RALS for benign gynecologic pathology. We selected a subset of patients who had surgery through the division of Minimally Invasive Gynecologic Surgery at Brigham and Women's Hospital in 2010 and administered a mixed-mode survey. The questionnaire was completed by 99 of 251 potential respondents (39\%). Nine patients (10\%) had RALS and 90 patients (90\%) had CLS. Demographics and type of surgery did not differ between the two groups. The types of procedures performed included surgery for endometriosis (10\%), hysterectomy (59\%), myomectomy (7\%), and oophorectomy (21\%). Patients who had RALS were more likely to have had more than one type of prior surgery (44\% versus $12 \% ; P=0.036)$ and to have learned about RALS through a recommendation from their doctor (78\% versus $28 \% ; P=0.004$ ). Both the RALS and CLS groups were likely to recommend their respective surgical approach (mean [SEM] Likert scale score, 3.78 [0.43] versus 3.72 [0.15]; $P=0.895$ ). Both the RALS and CLS groups tended to prefer CLS if RALS led to higher cost (mean [SEM] Likert scale score, 3.13 [0.52] versus 3.37 [0.14]; $P=0.656$ ), or longer duration of surgery (mean [SEM] Likert scale score, 3.13 [0.35] versus 3.51 [0.13]; $P=0.895$ ). RALS and CLS have comparable outcomes in benign gynecology. Patients at our institution do not have a bias toward either approach, though they tend to prefer the approach that will lead to lower cost and shorter operative time.
\end{abstract}

Keywords: Robotic surgery; Laparoscopy; Gynecology; Patient perceptions

\section{Introduction}

Robotic-assisted laparoscopic surgery (RALS) has been widely adopted in the surgical fields. In 2009, approximately 205,000 surgeries were performed robotically worldwide [1]. RALS has been commonly utilized in gynecologic surgery for the performance of hysterectomy, myomectomy, sacrocolpopexy, and endometriosis resection [2]. In the first quarter of 2010, RALS was used to perform nearly $10 \%$ of hysterectomies in the United States, and in hospitals that owned a da Vinci Robotic platform, RALS was used to perform $25 \%$ of hysterectomies [3]. Studies indicate that robot-assisted laparoscopy has the potential to shorten the laparoscopic learning curve for novice surgeons, allowing the accomplishment of advanced laparoscopic tasks, such as suturing and knot tying, at rates comparable to more experienced surgeons [4]. As a result, proponents suggest that RALS is an enabling technology, allowing patients to have a minimally invasive surgical (MIS) approach in cases that would otherwise be completed by laparotomy [5]. However, when compared to conventional laparoscopic surgery (CLS), no study has conclusively shown a benefit in patient outcomes, and some studies have associated the use of RALS with higher cost and longer operative time [3,6,7].

One factor potentially influencing the dissemination of RALS is that the marketing strategy for robotic surgery has been predominantly web-based, making it highly accessible to patients [8]. No study to date has evaluated whether direct-to-consumer marketing influences women pursuing minimally invasive surgery for benign gynecologic disease. 
The aim of this study was to evaluate the factors women consider in the decision to pursue minimally invasive surgery for benign gynecologic pathology. In particular, we aimed to focus on participant perceptions about minimally invasive surgery, prior knowledge of robotic-assisted laparoscopy, and importance placed on time under anesthesia and cost [9].

\section{Methods}

After obtaining institutional review board consent from our institution, we developed an anonymous 19-question mixed-mode survey designed to explore factors women consider when deciding to pursue minimally invasive gynecologic surgery. The survey included questions about demographic characteristics, medical history, patients' background knowledge, perceived benefits of robotic-assisted laparoscopic surgery, and preferences with regards to surgical approach when considering cost and duration under anesthesia. Questions were asked using a multiple-choice format as well as a Likert-type ranking format.

We identified patients who had surgery through the Minimally Invasive Gynecologic Surgery (MIGS) Division at Brigham and Women's Hospital (BWH) in the year 2010. We identified 15 patients who had robot-assisted laparoscopic surgery and 940 patients that had conventional laparoscopic procedures. In the absence of baseline data information on which to base a power calculation, a convenience sample was utilized. All patients who had robot-assisted laparoscopic surgery were invited to participate. Given the large number of patients who had conventional laparoscopic procedures compared to robotic procedures, a random sample of patients who underwent CLS was selected for participation. The CLS group was stratified by surgeon, race, age, and procedure and then assigned a number one through four using a random number generator. All patients who had CLS that were assigned the number one were selected to participate.

The initial surveys were sent on March 13, 2012, May 24, 2012, and June 22, 2012. We mailed selected participants an introductory letter, the questionnaire, a link to an on-line version of the questionnaire, and a self-addressed postmarked envelope to facilitate the return of the questionnaire. A second mailing was sent out two weeks after the initial mailing. Patients could either complete the questionnaire on-line or on paper. The questionnaires were anonymous and no personal identifiers were collected. Informed consent was implied by completion and submission of the survey [10].

For data analysis, we used the Fischer exact test for categorical variable, Students T test for continuous variables, and Wilcoxon rank sums tests for non-normally distrubuted continuous variables. All p values were 2 -sided, and those less than .05 were considered to indicate statistical significance. All analyses were performed with SAS version 9.2 (SAS Institute, Inc., Cary, NC) [11].

\section{Results}

The questionnaire was completed by 99 of 251 potential respondents, for a study sample response rate of $39.4 \%$. Nine respondents (10.0\%) had robot-assisted laparoscopic surgery, and 90 (90.0\%) had conventional laparoscopic surgery. Age, race, income, education, and parity did not vary between those who had robotic assisted surgery and those who had conventional laparoscopy (Table 1). Women who had RALS were more likely than women who had CLS to report a prior laparoscopic surgery (88.9\% versus $37.8 \% ; P=0.004)$ and more than one type of prior abdominal surgery $(44.4 \%$ versus $12.2 \% ; P=0.036)$. The types of surgeries performed by surgeons from the BWH MIGS division included surgical treatment of endometriosis (10.1\%), hysterectomy (59.6\%), myomectomy (7.0\%), oophorectomy (21.2\%) and other procedures (14.1\%), and did not vary significantly between the two groups (Table 1). Other surgeries included five urogynecological suspension procedures; two mesh excisional procedures, two lysis of adhesions procedures, one tubal procedure, and one resection of a pelvic mass.

When asked about prior knowledge of RALS, patients who had RALS were not more likely than patients who had CLS to report they learned of the approach through an advertisement $(22.2 \%$ versus $33.3 \% ; P=1.000)$, but were more likely to report that they had learned about RALS through a recommendation from their doctor $(77.8 \%$ versus $27.8 \%$; $P=0.004)$. Patients who had RALS were not more likely than women who had CLS to report that they selected their doctor because he or she performed robotic assisted surgery (22.2\% versus $16.7 \% ; P=0.66)$.

When women were asked about which minimally invasive approach they perceived to be the safest, $16.3 \%$ selected robotic surgery, $15.3 \%$ selected conventional laparoscopy, $17.3 \%$ selected equal safety between robotic-surgery and conventional laparoscopy, and $51.0 \%$ selected that they were unsure of which was the safest approach. There was no significant difference in response to the perceived safest approach between women who RALS and women who underwent CLS $(P=0.06)$.

When women who had robotic-assisted laparoscopic surgery were asked what they perceived to be the greatest advantage of that approach, 33.3\% selected shorter recovery time, 33.3\%, selected avoidance of laparotomy, 16.7\% selected use of technology, and $16.7 \%$ selected that they did not perceive any benefit.

Seventy-five percent of women who had RALS and $86.9 \%$ of women who had CLS reported that they would opt to have RALS despite higher cost if it was associated with fewer complications $(P=0.315)$. Similarly, $85.7 \%$ of women who had RALS and $81.9 \%$ of women who had CLS reported that they would opt to have RALS despite longer operating room time (ORT) if it was associated with fewer complications (Table 2). 


\begin{tabular}{|c|c|c|c|}
\hline & RALS & CLS & p-value \\
\hline & $N=9$ & $N=90$ & \\
\hline \multicolumn{4}{|c|}{$\mathrm{Age}^{\dagger}$} \\
\hline Mean (SD) & $46.33(6.81)$ & $48.65(9.93)$ & 0.373 \\
\hline \multicolumn{4}{|c|}{ Race/Ethnicity ${ }^{\dagger}$} \\
\hline Caucasian & $7(77.7 \%)$ & $72(81.0 \%)$ & 0.625 \\
\hline Hispanic & $1(11.1 \%)$ & $2(2.3 \%)$ & \\
\hline Black & $1(11.1 \%)$ & $9(10.0 \%)$ & \\
\hline Asian & $0(0 \%)$ & $3(3.4 \%)$ & \\
\hline Other & $0(0 \%)$ & $3(3.4 \%)$ & \\
\hline \multicolumn{4}{|c|}{ Education $^{\dagger}$} \\
\hline High school & $1(11.1 \%)$ & $11(12.5 \%)$ & 0.646 \\
\hline College & $6(66.7 \%)$ & $48(54.6 \%)$ & \\
\hline Graduate School & $2(22.2 \%)$ & $29(33.0 \%)$ & \\
\hline \multicolumn{4}{|c|}{ Parity $^{\dagger}$} \\
\hline Median (Range) & $1(0-4)$ & $2(0-6)$ & 0.915 \\
\hline \multicolumn{4}{|c|}{ Income $^{\dagger}$} \\
\hline$<\$ 50 \mathrm{~K}$ & $2(22.2 \%)$ & $11(13.6 \%)$ & 0.850 \\
\hline$\$ 51 \mathrm{~K}-\$ 100 \mathrm{~K}$ & $2(22.2 \%)$ & $28(34.6 \%)$ & \\
\hline$\$ 101 \mathrm{~K}-\$ 150 \mathrm{~K}$ & $3(33.3 \%)$ & $21(25.9 \%)$ & \\
\hline$>\$ 151$ & $2(22.2 \%)$ & $21(25.9 \%)$ & \\
\hline \multicolumn{4}{|c|}{ Prior abdominal surgery } \\
\hline No prior surgery & $1(11.1 \%)$ & $34(37.8 \%)$ & 0.153 \\
\hline Prior CLS & $8(88.9 \%)$ & $34(37.8 \%)$ & 0.004 \\
\hline Prior RALS & $0(0 \%)$ & $2(2.2 \%)$ & 1.000 \\
\hline Prior open surgery & $4(44.4 \%)$ & $30(33.3 \%)$ & 0.489 \\
\hline More than one type of surgery & $4(44.4 \%)$ & $11(12.2 \%)$ & 0.036 \\
\hline \multicolumn{4}{|c|}{ Procedure $^{*}$} \\
\hline Oophorectomy & $4(44.4 \%)$ & $17(18.9 \%)$ & 0.092 \\
\hline Endometriosis & $1(11.1 \%)$ & $9(10.0 \%)$ & 1.000 \\
\hline Hysterectomy & $6(58.9 \%)$ & $53(66.7 \%)$ & 0.736 \\
\hline Myomectomy & $1(11.1 \%)$ & $6(6.7 \%)$ & 0.498 \\
\hline Cancer & $0(0 \%)$ & $0(0 \%)$ & NA \\
\hline Other & $2(22.2 \%)$ & $12(13.3 \%)$ & 0.611 \\
\hline \multicolumn{4}{|c|}{ Selected doctor based on ability to perform RALS } \\
\hline Yes & $2(22.2 \%)$ & $15(16.7 \%)$ & 0.655 \\
\hline No & $7(77.8 \%)$ & $73(83.0 \%)$ & \\
\hline \multicolumn{4}{|c|}{ Prior knowledge of RALS } \\
\hline Advertisement & $2(22.2 \%)$ & $30(30.0 \%)$ & 0.611 \\
\hline
\end{tabular}




\begin{tabular}{|c|c|c|c|}
\hline \multirow{2}{*}{} & $\boldsymbol{R A L S}$ & $\boldsymbol{C L S}$ & $\boldsymbol{p}^{\text {-value }} \boldsymbol{*}^{*}$ \\
\cline { 2 - 4 } & $\boldsymbol{N}=\mathbf{9}$ & $\boldsymbol{N}=\mathbf{9 0}$ & \\
\hline Word of mouth & $2(22.2 \%)$ & $20(22.2 \%)$ & 1.000 \\
\hline Recommendation from a doctor & $7(77.8 \%)$ & $25(27.8 \%)$ & 0.005 \\
\hline No prior knowledge of RALS & $1(11.1 \%)$ & $24(26.7 \%)$ & 0.442 \\
\hline
\end{tabular}

${ }^{*} p$-values from Fisher's exact tests for categorical variables, t-tests for continuous variables, and Wilcoxon rank sums tests for discrete variables 'Some patients had missing data. The total missing for each variable is age $(n=1)$, race/ethnicity $(n=1)$, education $(n=2)$, parity $(n=1)$, income $(n=9)$, selected doctor based on ability to perform RALS $(n=2)$

*Categories are not mutually exclusive

Table 1: Patient demographic and surgical characteristics by robot-assisted laparoscopic surgery (RALS) versus conventional laparoscopic surgery (CLS)

\begin{tabular}{|c|c|c|c|}
\hline \multirow{2}{*}{ Patient perceptions } & RALS & CLS & $p$-value ${ }^{*}$ \\
\hline & $\mathrm{N}=9$ & $\mathrm{~N}=90$ & \\
\hline \multicolumn{4}{|c|}{ Prefer RALS over CLS despite higher cost if fewer complications ${ }^{\dagger}$} \\
\hline Yes & $6(75.0 \%)$ & $73(86.9 \%)$ & 0.315 \\
\hline No & $2(25.0 \%)$ & $11(13.1 \%)$ & \\
\hline \multicolumn{4}{|c|}{ Prefer RALS over CLS despite longer ORT if fewer complications ${ }^{\dagger}$} \\
\hline Yes & $6(85.7 \%)$ & $68(81.9 \%)$ & 1.000 \\
\hline No & $1(14.3 \%)$ & $15(18.1 \%)$ & \\
\hline
\end{tabular}

ORT = operating room time

${ }^{\dagger}$ Some patients had missing data. The total missing for each variable is prefer RALS over CLS despite higher cost if fewer complications $(\mathrm{n}=7)$, Prefer RALS over CLS despite longer ORT if fewer complications $(\mathrm{n}=9)$

Table 2: Patient perceptions about minimally invasive surgical approaches by robot-assisted laparoscopic surgery (RALS) versus conventional laparoscopic surgery (CLS)

Both the RALS and CLS groups were likely to recommend their respective surgical approach to a family member (mean [SEM] Likert-scale score, 3.50 [0.59] versus 3.44 [0.11]; $P=0.51$ ). Similarly, both women who had RALS and women who had CLS tended to prefer the use of conventional laparoscopy over RALS in the case that the use of RALS lead to higher cost (mean [SEM] Likert scale score, 3.15 [0.52] versus 3.45 [0.13]; $P=0.48$ ), or longer duration of surgery/general anesthesia (mean [SEM] Likert scale score, $3.15[0.52]$ versus $3.45[0.13] ; P=0.28$ ) (Figure 1).

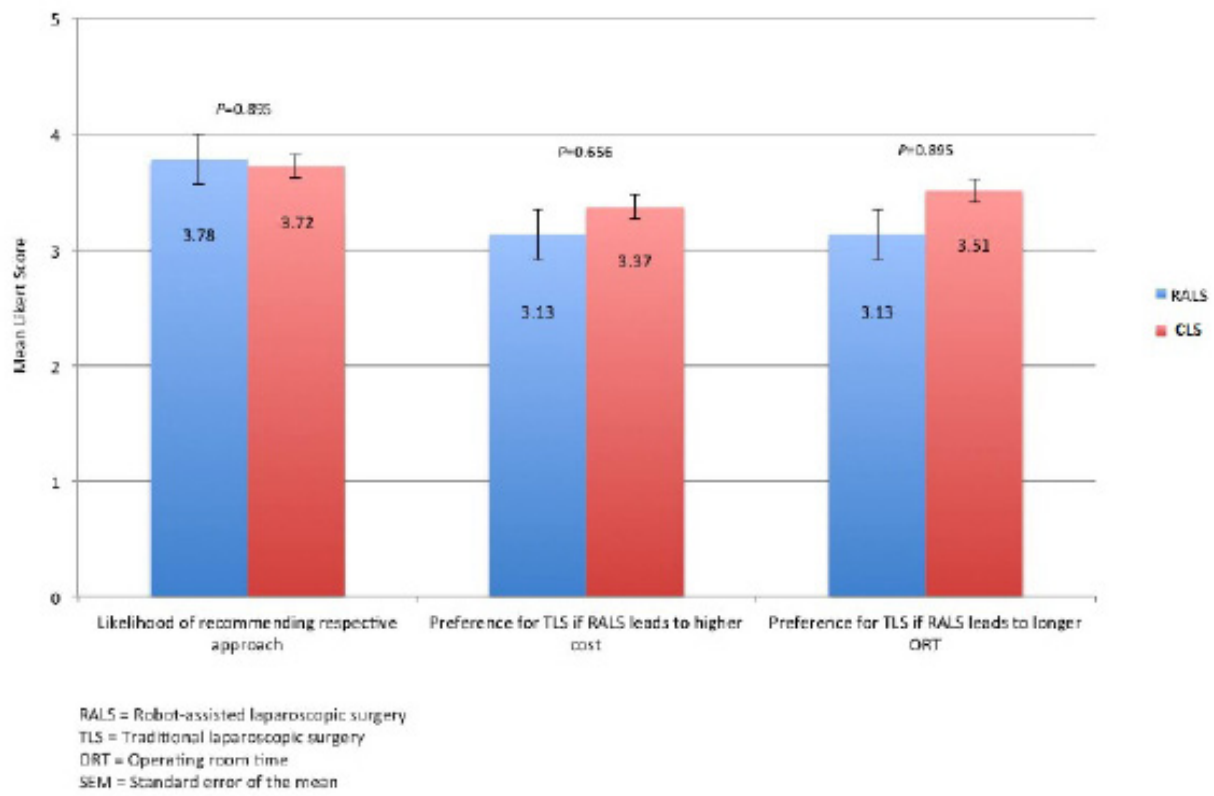

Figure 1: Patient preferences by surgical approach. Questions were scored on a 5-point Likert scale. For each question, mean values were calculated to generate a single response score. For the question regarding likelihood of recommending respective approach, the score ranged from 1, which indicated very unlikely, to 5, which indicated very likely. For the questions regarding preference for approach if RALS leads to higher cost or longer ORT, the score ranged form 1, which indicated strong preference for RALS, to 5, which indicated a strong preference for TLS. Error bar indicate SEM 
Journal of Gynecology Research

\section{Discussion}

The present study evaluated patients' perceptions about both RALS and CLS. Patients tended to be well educated and from a middle-income demographic group. Participants from both the CLS and RALS groups tended to prefer CLS if RALS were to lead to higher cost or longer operative time. However, patients also reported that they were willing to accept higher cost and longer operative time if RALS leads to fewer complications. Most women reported they were uncertain about which was the safest approach between RALS and CLS.

One prior study in patients undergoing colorectal surgery suggests an influence of marketing on patients selecting a robotic approach [12]. In the present study, only $32 \%$ of participants reported having heard of RALS through an advertisement and only $17 \%$ reported selecting their doctor based on their ability to perform RALS. Women who had RALS were not more likely to have heard of RALS through an advertisement. They were most likely to have learned about RALS through the recommendation of their doctor and were not more likely to select their doctor due to their ability to perform RALS. Notably, patients who had RALS were more likely to report more that one type of abdominal surgery, thus potentially increasing the complexity of their surgery and influencing their doctor's recommendation to use RALS as the approach to their surgery.

It is unclear if the limited marketing influence in our patient population is exclusively due to patient guided factors or is resultant from the Pharmaceutical and Medical Device Manufacturer Code of Conduct that is strictly implemented in the state of Massachusetts. This code of conduct restricts sales and marketing activities and limits interactions between physicians and the manufacturers of pharmaceutical and medical devices [13]. It is possible that in states with less restrictive policies, more influence from marketing would be present.

The authors recognize several limitations of this research study. The sample size is small, thus compromising the statistical power to see a difference between women who had RALS and those who had CLS. A larger study with a more balanced representation of women who have had RALS and CLS may be able to provide a more complete description on factors that patients find important when pursuing MIGS for benign gynecologic pathology. Second, our study population was predominantly well educated and from a middle-class background. A more diverse patient population is necessary to enhance the generalizability of these results. Third, the Brigham and Women's Hospital (BWH) MIGS division practices predominantly CLS and thus, it is possible patients preferring RALS were less likely to present as patients to the BWH MIGS practice, thus biasing our findings. Finally, it was not possible to test the survey tool for validity and reliability prior to implementation due to restrictions in time and funding. The major strength of our study is that despite the small sample size, we were able to demonstrate the opinion of women who had two different MIGS approaches. Both groups of women responded that they were likely to recommend their respective approach, and though their responses indicated that though cost and operative time played a role in which approach they favored, they placed importance on minimizing complications above cost and operative time.

The results of the study raise several questions that should be addressed in the future. It is likely that diverse populations will have varying perspectives about minimally invasive surgery and the factors they prioritize when making a decision about a surgical approach. Another question to address is patient preferences with regards to MIGS approaches based on their particular surgeon's expertise. Our study indicates that patients prioritize reduction of complications and their willingness to undergo a surgical approach that is less costly would likely change if the outcomes are not equivalent to the surgeons preferred approach.

As the use of RALS continues to increase worldwide, there is a growing need for timely and rigorous evidence to determine which patients can benefit from RALS. In addition, more information is needed about factors that influence patients when pursuing a MIGS approach.

\section{Conclusion}

Women undergoing MIGS at our institution do not seem to have a bias towards either RALS or CLS. Both groups are likely to recommend their respective approach. Women who underwent RALS report that they did so at the recommendation of their doctor and are not more likely to select their doctor because they perform RALS, or to report that they have heard of RALS through an advertisement. These findings highlight the role of surgeon preference for determining the surgical approach. Both groups prioritize decreased complications over cost and time in the operating room. However, if outcomes are equivalent, patients tend to prefer the approach that minimizes cost and operative time. Understanding the factors that women value when pursuing MIGS for benign gynecologic pathology is essential to provided comprehensive counseling about their surgical options. Future research is needed to elucidate the benefits of RALS and the factors that influence patients when pursuing a MIGS approach to treatment of benign gynecological disease.

\section{Acknowledgement}

This work was funded by the Brigham and Women's Department of Obstetrics and Gynecology Expanding the Boundaries Grant. 


\section{Reference}

1. Barbash GI, Glied SA (2010) New technology and health care costs--the case of robot-assisted surgery. N Engl J Med 363: 701-4.

2. Sinha R, Sanjay M, Rupa B, Kumari S (2015) Robotic surgery in gynecology. J Minim Access Surg 11: 50-9.

3. Wright JD, Ananth CV, Lewin SN, Burke WM, Lu YS, et al. (2013) Robotically assisted vs laparoscopic hysterectomy among women with benign gynecologic disease. JAMA 309: 689-98.

4. Chandra V, Nehra D, Parent R, Woo R, Reyes R, et al. (2010) A comparison of laparoscopic and robotic assisted suturing performance by experts and novices. Surgery 147: 830-9.

5. Advincula AP, Wang K (2009) Evolving role and current state of robotics in minimally invasive gynecologic surgery. J Minim Invasive Gynecol 16: 291-301.

6. Liu H, Lawrie TA, Lu D, Song H, Wang L, et al. (2014) Robot-assisted surgery in gynaecology. Cochrane Database Syst Rev 12: CD011422.

7. Nieboer TE, Johnson N, Lethaby A, Tavender E, Curr E, et al. (2006) Surgical approach to hysterectomy for benign gynaecological disease. Cochrane Database Syst Rev 19: CD003677.

8. Schiavone MB, Kuo EC, Naumann RW, Burke WM, Lewin SN, et al. (2012) The commercialization of robotic surgery: unsubstantiated marketing of gynecologic surgery by hospitals. Am J Obstet Gynecol 207: 174.e1-7.

9. Alkhateeb S, Lawrentschuk N (2011) Consumerism and its impact on robotic-assisted radical prostatectomy. BJU Int 108: 1874-8.

10. Mirkin JN, Lowrance WT, Feifer AH, Mulhall JP, Eastham JE, et al. (2012) Direct-to-consumer Internet promotion of robotic prostatectomy exhibits varying quality of information. Health Aff 31: 760-9.

11. Jin LX, Ibrahim AM, Newman NA, Makarov DV, Pronovost PJ, et al. (2011) Robotic surgery claims on United States hospital websites. J Healthc Qual 33: 48-52.

12. Dixon PR, Grant RC, Urbach DR (2015) The impact of marketing language on patient preference for robot-assisted surgery. Surg Innov 22: 15-19.

13. Massachusetts Department of Health and Human Services (2014). Background information about the code of conduct.

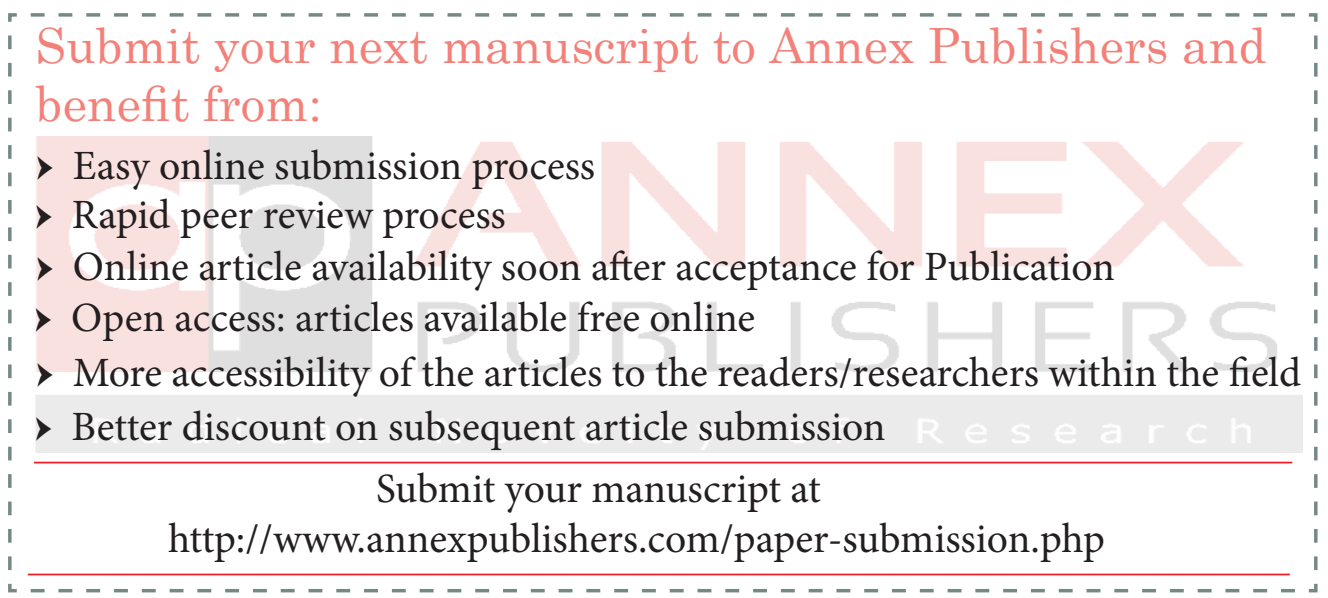

